OPEN ACCESS

Edited by:

Jean-François Cloutier, Montreal Neurological Institute and Hospital, McGill University, Canada

Reviewed by: Satoshi Ogawa, Monash University Malaysia, Malaysia Suresh Jesuthasan, Nanyang Technological University, Singapore

${ }^{*}$ Correspondence: Hae-Chul Park hcpark67@korea.ac.kr

${ }^{\dagger}$ These authors have contributed equally to this work

Received: 23 April 2019 Accepted: 26 July 2019 Published: 14 August 2019

Citation: Jeong I, Kim E, Seong JY and Park H-C (2019) Overexpression of Spexin 1 in the Dorsal Habenula Reduces Anxiety in Zebrafish. Front. Neural Circuits 13:53. doi: 10.3389/fncir.2019.00053

\section{Overexpression of Spexin 1 in the Dorsal Habenula Reduces Anxiety in Zebrafish}

\author{
Inyoung Jeong ${ }^{1 \dagger}$, Eunmi Kim ${ }^{1 \dagger}$, Jae Young Seong ${ }^{2}$ and Hae-Chul Park ${ }^{1 *}$ \\ ${ }^{1}$ Department of Biomedical Sciences, College of Medicine, Korea University, Ansan, South Korea, ${ }^{2}$ Department of Biomedical \\ Sciences, Korea University, Seoul, South Korea
}

Spexin (SPX) is an evolutionarily conserved neuropeptide that is expressed in the mammalian brain and peripheral tissue. Two orthologs are present in the teleost, SPX1 and SPX2. SPX1 is involved in reproduction and food intake. Recently, SPX1 neurons have been found to be located in the specific nuclei of dorsal habenula $(\mathrm{dHb})$ and to project into the interpeduncular nucleus (IPN), in which galanin receptor $2 a / 2 b(G A L R 2 a / 2 b)$ expression was also observed. This indicates that habenula SPX1 neurons may interact with GALR2a/2b in the IPN; however, the function of SPX1 in the $\mathrm{dHb}-I P N$ neuronal circuit remains unknown. To determine the role of SPX1 in the dHb-IPN neural circuit, we generated transgenic zebrafish overexpressing SPX1 specifically in the $\mathrm{dHb}$. We found that transgenic zebrafish overexpressing SPX1 in the $\mathrm{dHb}$ had anxiolytic behaviors compared with their wildtype siblings. Furthermore, quantitative PCR revealed that mRNA expression of galr2a and galr2b in the IPN and serotonin-related genes in the raphe was upregulated in the brains of transgenic zebrafish. Taken together, our data suggest that SPX1 function in the dHb-IPN neural circuits is implicated in the regulation of anxiety behaviors via modulation of the serotoninergic system in zebrafish.

Keywords: zebrafish, anxiety, spexin 1, dorsal habenula, serotonergic system

\section{INTRODUCTION}

Spexin (also known as SPX, NPQ, and C12ORF39) is a secreted neuropeptide that is mainly expressed in the brain, pancreas, kidney, liver, ovary, and adrenal glands of mammals and fish (Mirabeau et al., 2007; Sonmez et al., 2009; Porzionato et al., 2010; Rucinski et al., 2010; Liu et al., 2013; Wong et al., 2013). The functional mature peptide domain of SPX consists of 14 amino acids (NWTPQAMLYLKG ${ }^{\mathrm{A}} / \mathrm{T} \mathrm{Q}$ ), which have multiple physiological functions, including adrenocortical cell proliferation, cardiovascular function, urine flow, nociception, reproduction, and food intake (Rucinski et al., 2010; Toll et al., 2012; Liu et al., 2013; Wong et al., 2013; Ma et al., 2018). Zebrafish have two SPX orthologs, SPX1 and SPX2, because of two-round whole-genome duplication (Kim et al., 2014). The amino acid sequence of zebrafish SPX1 differs from that of mammalian SPX by only one amino acid at the mature peptide domain $(\mathrm{A} \leftrightarrow \mathrm{T})$, suggesting that $\mathrm{SPX}$ functions might be evolutionarily conserved in zebrafish (Liu et al., 2013). 
The habenula is a pair of small nuclei, which is involved in pain, stress, fear/anxiety, sleep, and reward behaviors in the brain (Hikosaka, 2010). The habenula consists of the medial habenula $(\mathrm{MHb})$ and lateral habenula ( $\mathrm{LHb})$ in mammals. The $\mathrm{MHb}$ is a component of the evolutionarily conserved dorsal diencephalic conduction system, which is essential for anxiety and mood regulation (Yamaguchi et al., 2013; Hsu et al., 2016). The dorsal habenula $(\mathrm{dHb})$ in zebrafish is the ortholog of the $\mathrm{MHb}$ in mice (Amo et al., 2010) and dHb-interpeduncular nucleus (IPN) circuitry is also involved in fear and anxiety responses (Agetsuma et al., 2010; Mathuru and Jesuthasan, 2013). We recently demonstrated that SPX1 neurons are located in the specific nuclei of $\mathrm{dHb}$, which regulates fear and anxiety, and $\mathrm{dHb}$ SPX1 neurons project their axons to the galanin receptor $2 \mathrm{a} / 2 \mathrm{~b}$ (GALR2a/2b) neurons in the IPN of the zebrafish brain (Pandey et al., 2018; Kim et al., 2019). However, there is no direct evidence that $\mathrm{dHb}$ SPX1 function is linked to anxiety.

To assess the function of SPX1 in the $\mathrm{dHb}$, we first generated $\quad \mathrm{Tg}$ (gpr151:gal4vp16); $\mathrm{Tg}$ (5xuas:spx1:p2a-mCherr y) zebrafish, which overexpressed SPX1 in the $\mathrm{dHb}$, using the GAL4XUAS transactivator system. Subsequently, we analyzed the anxiety response using the novel tank test and expression of galr $2 \mathrm{a} / 2 \mathrm{~b}$ and serotonin-related genes using quantitative RT-PCR in $T g(g p r 151: g a l 4 v p 16) ; \operatorname{Tg}(5 x u a s: s p x 1: p 2 a-$ mCherry) zebrafish.

\section{MATERIALS AND METHODS}

\section{Zebrafish Lines}

All experimental procedures were approved by the Korea University Institutional Animal Care \& Use Committee (IACUC) and performed in accordance with Animal experiment guidelines of Korea National Veterinary Research and Quarantine Service. Tg(gpr151:gal4vp16; Chou et al., 2016) and $T g$ (5xuas:spx1:p2a-mCherry) zebrafish were used in this study. All zebrafish were $6-8$ month-old male adults, raised in a 14:10 light:dark cycle at $28.5^{\circ} \mathrm{C}$.

\section{Plasmid Construct and Generation of Transgenic Zebrafish}

To generate $\operatorname{Tg}$ (5xuas:spx1:p2a-mCherry) zebrafish, we performed PCR to amplify the spx1 ORF from cDNA using the following forward and reverse primers containing attB1 and attB2 sites: spx1 forward, 5'-GGGGACAAGTTTGTACAAAA AAGCAGGCTCCATGA AAGATTTAAGGACTCTTGCGGC G-3'; spx1 reverse, 5'-GGGGACCACTTTGTACAAGA AAGC TGGGTCCCAGTACGGCTCGTCTTCATCTCTG-3'. The PCR product containing attB sites was cloned into a middle entry vector using the BP reaction of the gateway system (Invitrogen). In addition, we cloned the $5^{\prime}$-entry clone containing five tandem repeated uas ( $5 \mathrm{x} u a s$ ) sequences and the $3^{\prime}$-entry clone containing the mCherry fused viral 2A peptide (Kim et al., 2011). The multi-site gateway LR reaction was performed using LR II clonase with entry clones, according to the manufacturer's instruction (Invitrogen). To produce a stable transgenic zebrafish line, we injected the 5xuas:spx1:p2a-mCherry DNA into one-cell embryos. To screen for germ-line transmitted transgenic zebrafish, we mated the 5xuas:spx1:p2a-mCherry DNA-injected founder zebrafish with transgenic zebrafish containing gal4 and screened the progeny for mCherry expression. The obtained transgenic founder fish were crossed with wild-type zebrafish, and F1 transgenic zebrafish were raised to adulthood.

\section{Adult Brain Preparation}

For the histological assay, 6-8-month-old male adult zebrafish $(n=5)$ were anesthetized using $200 \mathrm{mg} / \mathrm{L}$ of ethyl 3-aminobenzoate methanesulfonate salt (MS222, Sigma), until cessation of movement. Trans-cardiac perfusions were performed using phosphate buffered saline (PBS). Subsequently, the fish's heads were decapitated and their brains were fixed in their entirety, using 4\% paraformaldehyde. For total RNA preparation, 6-8-month-old adult zebrafish were sacrificed using the abovementioned procedures, with the exception of trans-cardiac perfusion and fixation.

\section{Whole-Mount in situ RNA Hybridization and Immunohistochemistry}

To detect spx 1 and $g p r 151$ RNA transcripts, we used the antisense RNA probe against $s p x 1$ and gpr151. The spx1 and gpr151cloned vectors were linearized by restriction endonucleases (New England Biolabs) and the linearized vectors were transcribed using the dig-oxygenin labeling mixture (Roche). Whole-mount in situ RNA hybridization of the whole adult brains was performed as described previously (Thisse and Thisse, 2008). Subsequently, the whole-mounted RNA in situ hybridized brain was fixed in $4 \%$ paraformaldehyde. The fixed brains were embedded in $1.5 \%$ agar blocks containing 5\% sucrose and equilibrated in $30 \%$ sucrose solution. The frozen blocks were sliced into $16-\mu \mathrm{m}$ sections using a cryostat microtome and the transverse sectioned slices were mounted on glass slides. The sections were rinsed with PBS several times and enclosed within $75 \%$ glycerol by coverslips. Images were obtained from the brain sections of $\operatorname{Tg}(g p r 151: g a l 4 v p 16) ; \operatorname{Tg}(5 x u a s: s p x 1: p 2 a-$ $m$ Cherry) fish and their siblings. For immunohistochemical analysis, the sections were rinsed with PBS several times and then blocked with $2 \%$ bovine serum albumin with sheep serum. After the blocking reaction, the sections were treated with primary antibodies for $2 \mathrm{~h}$ at room temperature, washed for $2 \mathrm{~h}$ with PBS, and subsequently treated with the appropriate secondary antibodies for $2 \mathrm{~h}$ at room temperature. Images were obtained from the sections of the adult brains of $\operatorname{Tg}($ spx 1:mcherry-caax); $\operatorname{Tg}$ (pou4f1-hsp70l:gfp) fish. We used the following antibodies for immunohistochemistry studies: rabbit anti-mCherry/Dsred (1:500, Clontech, Cat. No. 632496) and chicken anti-GFP (1:200, Abcam, Cat. No. AB13970). Alexa Fluor 488 and 647-conjugated secondary antibodies were used for fluorescent detection of primary antibodies (1:1,000, Invitrogen, Cat. No. A-21244 and Abcam, Cat. No. ab96947).

\section{Novel Tank Test}

The novel tank test was performed between 13:00 and 18:00. We followed the protocol that has been described previously (Cachat et al., 2010) with 6-8 months zebrafish. For adaptation, fish were moved to the behavior room $2 \mathrm{~h}$ before the test began. 


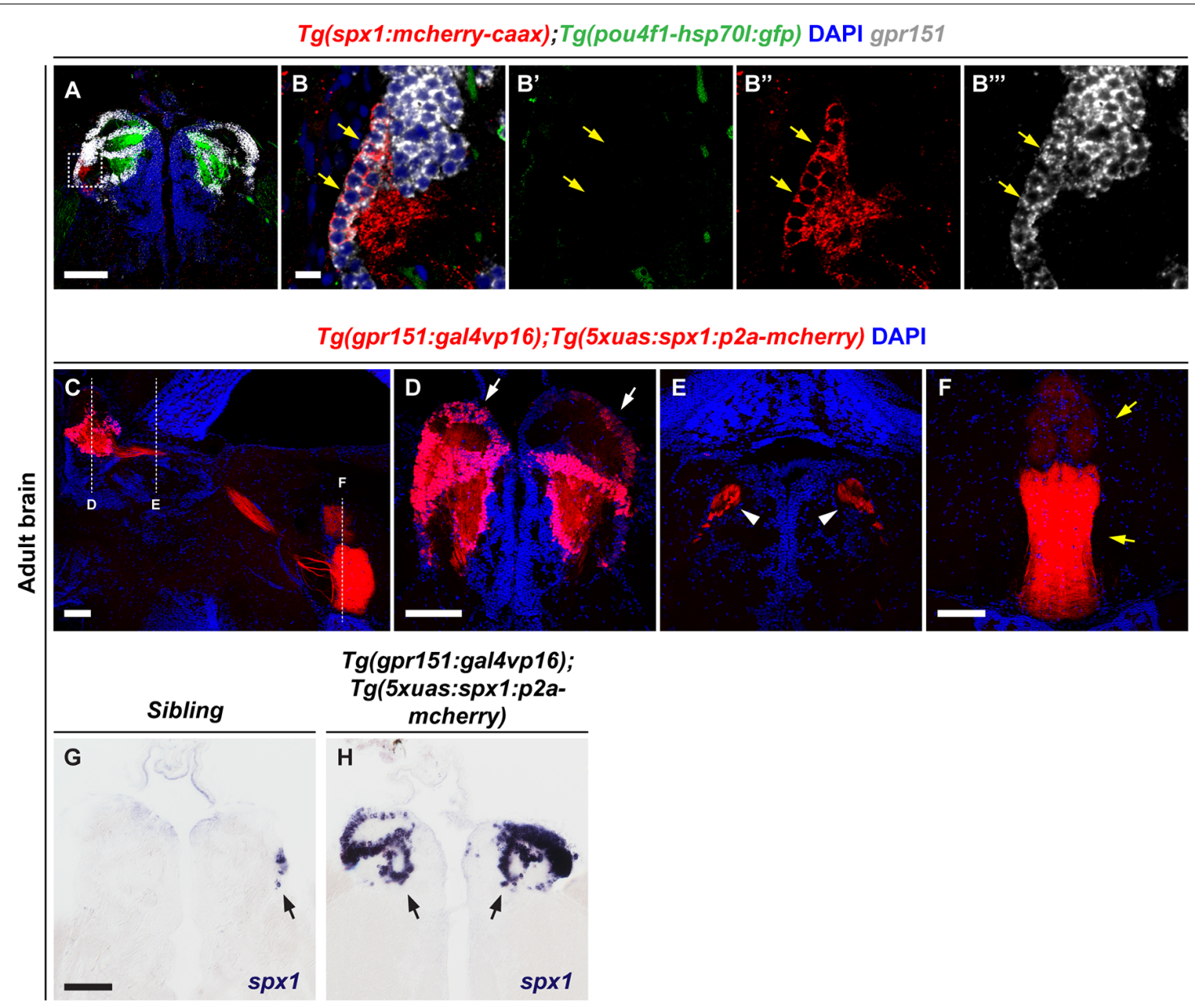

FIGURE 1 | Overexpression of spx1 in the dorsal habenula (dHb). Panels (A-B"') Transverse sections of the habenula of Tg(spx1:mCherry-CAAX); Tg(pou4f1hsp70l:gfp) zebrafish labeled by gpr151 RNA probe and DAPI. The dorsal aspect is toward the top. Panels (B-B"') are high magnification images of the box area in

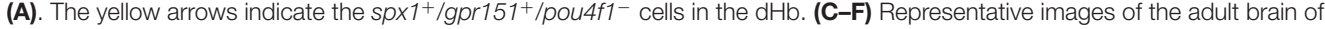
Tg(gpr151:gal4vp16);Tg(5xuas:spx1:p2a-mcherry) zebrafish stained with DAPI. (C) Sagittal section view. The dorsal aspect is to the top and the anterior is to the left. Each dotted line marks the transverse section views of every brain region. (D-F) Transverse section views. The dorsal aspect is toward the top. (D) The habenula. The white arrows indicate mCherry fluorescence in the $\mathrm{dHb}$. (E) The fasciculus retroflexus (FR). The white arrowheads indicate mCherry fluorescence in the FR. (F) The interpeduncular nucleus (IPN). The yellow arrows indicate mCherry fluorescence in the IPN. (G,H) Transverse section views of the habenula in Tg(gpr151:gal4vp16); Tg(5xuas:spx1:p2a-mcherry) zebrafish and their wildtype siblings labeled by whole-mount in situ RNA hybridization with an spx1 RNA probe The black arrows indicate spx1 mRNA expression in the habenula. Scale bar: $100 \mu \mathrm{m}$ in (A,C,D-H) and $10 \mu \mathrm{m}$ in (B-B'").

Adult behavior analyses were performed using a video camcorder (SONY). Behavior was recorded for $15 \mathrm{~min}$, and the recorded video files were analyzed using the automated tracking software, Ethovision XT 12 (Noldus). We analyzed the behavior for the first $10 \mathrm{~min}$ of each recording.

\section{Total RNA Preparation and Quantitative RT-PCR}

The whole-brain or IPN of the adult $T g(g p r 151: g a l 4 v p 16) ; T g$ (uas:gfp) and $\operatorname{Tg}(g p r 151: g a l 4 v p 16) ; \operatorname{Tg}(5 x u a s: s p x 1: p 2 a-m C h e r r y)$ zebrafish was isolated and the total RNA was extracted using TRIzol reagent (Invitrogen). Total RNA was extracted from adult brains using TRIzol reagent (Invitrogen). cDNA was synthesized from total RNA using a reverse transcription kit (ImProm-II ${ }^{\mathrm{TM}}$ Reverse Transcriptase, Promega). Quantitative
RT-PCR was performed using the LightCycler 96 Instrument (Roche). Each reaction mixture contained $2.5 \mu \mathrm{L}$ of $\mathrm{cDNA}$ as a template, $0.2 \mu \mathrm{M}$ forward and reverse primer, and $2 \times$ FastStart Essential DNA Green Master Mix (Roche). PCR was performed under the following conditions: $95^{\circ} \mathrm{C}$ for $10 \mathrm{~min}$, 40 cycles of $95^{\circ} \mathrm{C}$ for $10 \mathrm{~s}, 60^{\circ} \mathrm{C}$ for $10 \mathrm{~s}$, and $72^{\circ} \mathrm{C}$ for $10 \mathrm{~s}$. Gene expression of spexin 1 (spx1), galanin receptor $2 a$ (galr $2 a)$, galanin receptor $2 b$ (galr $2 b$ ), plasmacytoma expressed transcript 1 (pet1), tryptophan hydroxylase 2(tph2) and solute carrier family 6 , member $4 a($ slc6a4a) was verified using the primers described in Supplementary Table S1.

\section{Statistical Analysis}

All statistical analyses were performed using GraphPad prism 7 software (GraphPad Software). For two-group comparisons, 
A

Time after diving

$2 \mathrm{~min}$
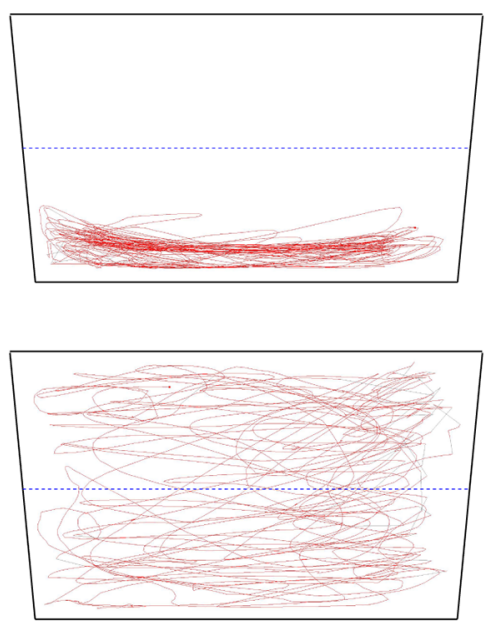

B

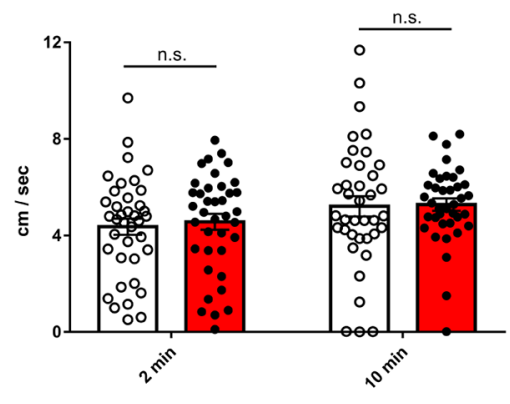

Time after diving

D

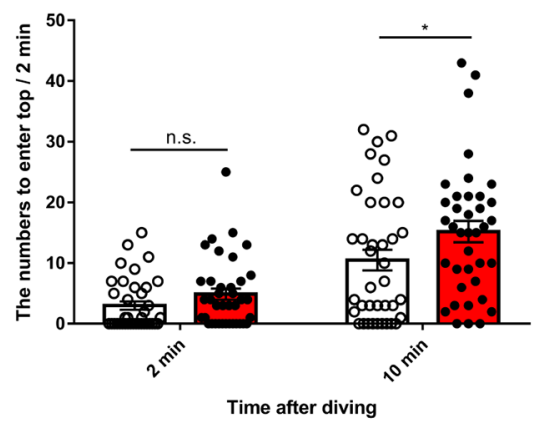

$10 \mathrm{~min}$

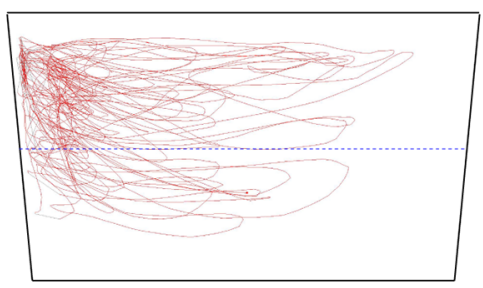

Sibling

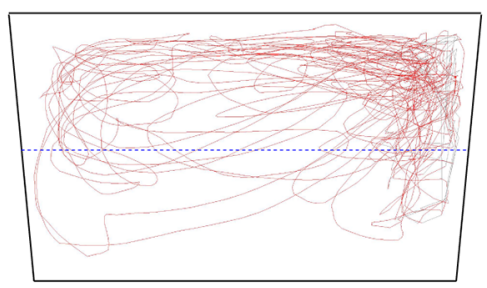

\section{Tg(gpr151:gal4vp16);}

Tg(5xuas:spx1:p2amcherry)
C

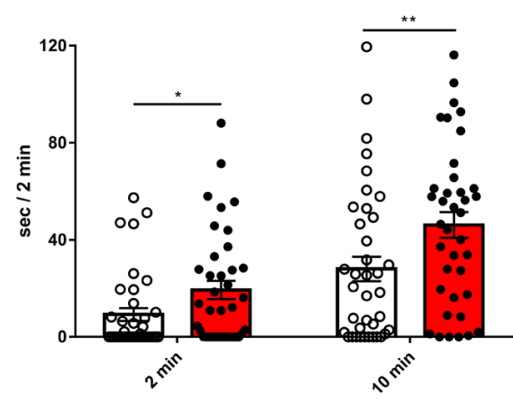

Time after diving

E

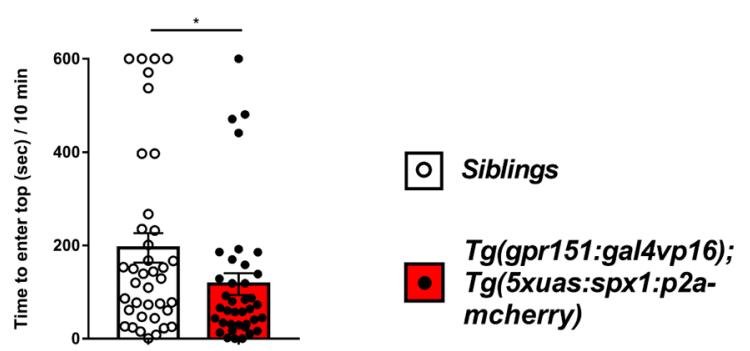

FIGURE 2 | Anxiety behavior of transgenic zebrafish overexpressing spx1 in the $\mathrm{dHb}$ and wildtype siblings. The novel tank test was used to assess the anxiety-related behaviors in adult Tg(gpr151:ga/4vp16);Tg(5xuas:spx1:p2a-mCherry) zebrafish (dHb-spx1 OV; $n=38$ ) and their wildtype siblings ( $n=38$ ). (A) Representative behavior-tracking results for 2 min at 2 and 10 min after diving. The black quadrangular boxes and blue dotted lines indicate the novel tanks and half lines of tanks. The red lines indicate the tracing lines of the moving fish. (B-D) Graph bars and error bars are expressed as mean \pm standard error of the mean (SEM). White or black dots in the graphs indicate individual data points. (B) The mean velocity at 2 min at 2 and 10 min after diving [2 min: siblings,

$4.365 \pm 0.3378 \mathrm{~cm} / \mathrm{s}$, dHb-spx1 OV, $4.565 \pm 0.3316 \mathrm{~cm} / \mathrm{s} ; 10 \mathrm{~min}$ (median): siblings, $4.816 \mathrm{~cm} / \mathrm{s}$; dHb-spx1 OV, $5.323 \mathrm{~cm} / \mathrm{s}$. n.s., not statistically significant]. The unpaired $t$-test was used for the 2-min analysis and the Mann-Whitney $U$-test for the 10-min analysis. (C) Time spent at the top half of the tank for 2 min, at 2 min and 10 min after diving [2 min (median): siblings, $0.02179 \mathrm{~s}$, dHb-spx1 OV, $11.56 \mathrm{~s} ; 10$ min (median): siblings, $19.5 \mathrm{~s}, \mathrm{dHb}-\mathrm{spx} 1 \mathrm{OV}, 48.88 \mathrm{~s} ;{ }^{*} p<0.05$,

${ }^{* *} p<0.005$, Mann-Whitney $U$-test]. (D) The number of fish that entered the top half of the tank for 2 min, at 2 min and 10 min after diving [2 min (median): siblings, 0.5; dHb-spx1 OV, 3.5. 10 min (median): siblings, 6.5; dHb-spx1 OV, 15.5; ${ }^{*} p<0.05$, n.s., not significant (Mann-Whitney U-test)]. (E) The latency to enter the top half of the tank. (Median: siblings, $134.4 \mathrm{~s}$; dHb-spx1 OV, 65.17 s; * $p<0.05$, Mann-Whitney U-test). 


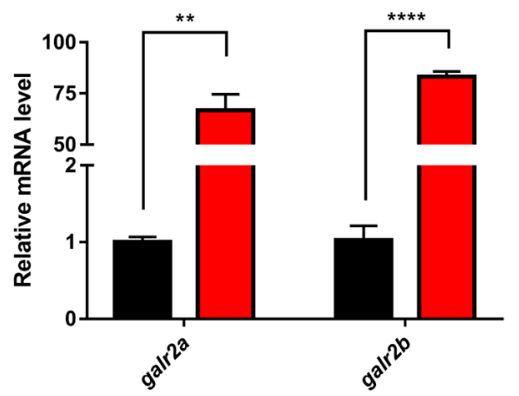

B

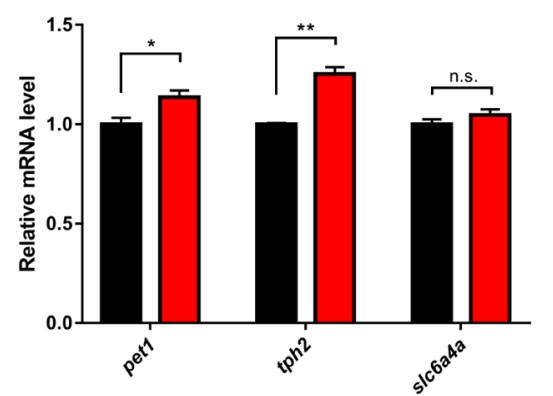

- Siblings

Tg(gpr151:gal4vp16);

Tg(5xuas:spx1:p2a-

mCherry)

FIGURE 3 | Upregulation of the galanin receptor 2a/2b (galr2a/b) and serotonin-related genes in the brain upon overexpression of spx 1 in the $\mathrm{dH}$. Quantitative RT-PCR in Tg(gpr151:gal4vp16);Tg(5xuas:spx1:p2a-mcherry) zebrafish (dHb-spx1 OV) and their wildtype siblings $\beta$-actin expression was used to normalize all samples, and data were acquired in triplicate. Data are represented as mean \pm SEM $(n=3)$. (A) Relative galr2a and galr2b mRNA expression in the IPN $(n=10$ per group): Galr2a: siblings, $1.003 \pm 0.06386$; dHb-spx1 OV, $66.78 \pm 7.778$. Galr2b: siblings, $1.03 \pm 0.1803$; dHb-spx1 OV, $83.17 \pm 2.561$. (B) Relative plasmacytoma expressed transcript 1 (pet1), tryptophan hydroxylase 2 (tph2), and slc6a4a mRNA expression in the brain ( $n=5$ per group): Pet1: siblings, $1 \pm 0.03215$; dHb-spx1 OV, $1.137 \pm 0.03383$. Tph2: siblings, $1 \pm 0.005774 ; \mathrm{dHb}-s p x 1 \mathrm{OV}, 1.253 \pm 0.03333$. S/c6a4a: siblings, $1 \pm 0.02517 ; \mathrm{dHb}-\mathrm{spx} 1 \mathrm{OV}, 1.047 \pm 0.02848\left[{ }^{*} p<0.05\right.$, ${ }^{* *} p<0.005,{ }^{* * * *} p<0.0001$ (Unpaired t-test), n.s., not significant].

Student's unpaired $t$-test was used when data were normally distributed, and the Mann-Whitney $U$-test was used when data were not normally distributed. Statistical significance was determined at $p<0.05$ (two-tailed).

\section{RESULTS}

\section{Generation of Transgenic Zebrafish Overexpressing spx1 in the Dorsal Habenula}

We previously demonstrated that $\mathrm{spx} 1$ is expressed in the specific nuclei of $\mathrm{dHb}$, and that $\mathrm{dHb}$ SPX1 neurons project to the IPN (Kim et al., 2019). To investigate the role of SPX1 in the $\mathrm{dHb}$, we first confirmed the respective expression patterns of spx1 and gpr151, which are expressed in the $\mathrm{dHb}$ (Broms et al., 2015), by fluorescent in situ RNA hybridization with gpr151, in the brains of $\operatorname{Tg}(s p x 1$ :mcherry-caax); $\operatorname{Tg}$ (pou4f1-hsp70l:gfp) adult zebrafish. Since $T g$ (pou4f1-hsp70l:gfp) zebrafish express poutfl:GFP in the medial division of dorsal habenula (dHbM; Aizawa et al., 2005), we could distinguish pou4fl:GFP ${ }^{-} \mathrm{dHb}$ in this transgenic line. We verified that gpr151 was expressed in the pou4fl: $\mathrm{GFP}^{-} \mathrm{dHb}$ as previously reported (Chou et al., 2016), and found that most of the spxl:mCherry ${ }^{+}$neurons co-express gpr151, although spx1:mCherry ${ }^{-}, g_{p r 151^{+}}$cells were also observed (Figures 1A-B). These data indicate that gpr151-expressing cells include most of the $s p x 1^{+}$cells in the $\mathrm{dHbL}$ area. To induce overexpression of SPX1 in the $\mathrm{dHb}$, we generated transgenic zebrafish that overexpress SPX1 in the $\mathrm{dHb}$ using the GAL4XUAS transactivator system. We produced Tg(5xuas:spx1:p2a-mCherry) zebrafish and crossed them with $\operatorname{Tg}($ gpr151:gal4vp16) zebrafish that express Gal4vp16 transactivator specifically in the $\mathrm{dHb}$ (Chou et al., 2016). Tg(gpr151:gal4vp16); $\operatorname{Tg}(5 x u a s: s p x 1: p 2 a-$ $m$ Cherry) zebrafish grew until adulthood without any morphological defects. To confirm the overexpression of SPX1 in the $\mathrm{dHb}$, we analyzed $s p \times 1:$ mCherry fluorescence in the brain of adult $T g$ (gpr151:gal4vp16); $T g(5 x u a s: s p x 1: p 2 a-m$ Cherry) zebrafish. Consistent with our previous observation that $\mathrm{dHb}$ SPX1 neurons project into the IPN, mCherry fluorescence was detected in the $\mathrm{dHb}$, fasciculus retroflexus (FR), and IPN in this transgenic zebrafish (Figures 1C-F). Whole-mount in situ RNA hybridization with $s p x 1$ in the brain of adult transgenic zebrafish and their wildtype siblings revealed that spxi was expressed in the limited area of the $\mathrm{dHb}$ of the wildtype siblings, as previously described (Kim et al., 2019), whereas increased spx1 expression was detected throughout the $\mathrm{dHb}$ (Figures $\mathbf{1 G}, \mathbf{H}$, Supplementary Figure S1). These data demonstrated that Tg(gpr151:gal4vp16); $\operatorname{Tg}$ (5xuas:spx1:p2a-mCherry) transgenic zebrafish overexpress $s p x 1$ in the $\mathrm{dHb}$.

\section{Overexpression of spx1 in the Dorsal Habenula Reduces Anxiety}

A previous study has shown that the $\mathrm{dHb}$ is associated with anxiety regulation in zebrafish (Mathuru and Jesuthasan, 2013). Thus, we hypothesized that SPX1 might be involved in anxiety regulation in the $\mathrm{dHb}$. To assess this, we performed the novel tank test (Cachat et al., 2010) using the Tg(gpr151: gal4vp16); $\operatorname{Tg}$ (5xuas:spx1:p2a-mCherry), which overexpress $s p x 1$ in the $\mathrm{dHb}$, and their wildtype siblings. Interestingly, we observed that transgenic zebrafish overexpressing spx1 spent more time at the top area of the tank after diving compared with their wildtype siblings (Figures 2A,C). In contrast, both transgenic and wildtype siblings showed similar locomotor activity (Figure 2B). Furthermore, transgenic zebrafish entered the top area of the tank more often at 10 min after diving compared with their siblings (Figures 2A,D). In addition, they have the tendency to visit the top area at $2 \mathrm{~min}$ after diving, which is more frequent than that in their siblings; however, this did not reach statistical significance (Figure 2D). Moreover, the latency to enter the top of the tank after diving decreased in the SPX1-overexpressing transgenic fish than wildtype siblings (Figure 2E). Taken together, these results 
indicated that transgenic fish overexpressing $s p x 1$ show increased anxiolytic behaviors compared with their wildtype siblings, suggesting that SPX1 is involved in the regulation of anxiety in the $\mathrm{dHb}$ in zebrafish.

\section{Anxiolytic Function of SPX1 Is Related With Upregulation of Serotonin-Related Gene Expression}

$\mathrm{dHb}$ neurons project into the IPN and the IPN is connected to the raphe, which is a part of the serotonergic system (Agetsuma et al., 2010; Okamoto et al., 2012; Chou et al., 2016). Therefore, we postulated that the serotonergic system might be modulated by the SPX1 neuronal pathway from the $\mathrm{dHb}$ and involved in the regulation of anxiety. To test this idea, we investigated whether overexpression of $s p x 1$ in $\mathrm{dHb}$ increased expression of its putative receptor, galr $2 a$, and galr $2 b$ (Kim et al., 2014). Quantitative RT-PCR with spx 1 and galr $2 a / 2 b$, showed that $s p x 1$ mRNA expression was 10 times higher in the brains of adult Tg(gpr151:gal4vp16); $\operatorname{Tg}$ (5xuas:spx1:p2a-mCherry) zebrafish compared with their wildtype siblings (Figure 3A). Interestingly, the expression of galr $2 a$, not galr $2 b$, was significantly increased in the brain of adult $T g(g p r 151: g a l 4 v p 16) ; T g(5 x u a s: s p x 1: p 2 a-$ $m$ Cherry) zebrafish compared with their wildtype siblings (Supplementary Figure S2). We further verified the expression of galr $2 a$ and galr $2 b$ with quantitative RT-PCR with cDNA isolated from the IPN and discovered that both, galr $2 a$ and galr $2 b$ expression was dramatically increased in the adult Tg(gpr151:gal4vp16); $\operatorname{Tg}$ (5xuas:spx1:p2a-mcherry) zebrafish, when compared with their wildtype siblings (Figure 3A). This suggested that increased SPX1 resulted in an increase in its putative receptor, GALR2a and GALR2b. Next, we examined the mRNA expression of serotonin-related genes in the raphe of adult $T g$ (gpr151:gal4vp16); $T g$ (5xuas:spx1:p2a-mCherry) zebrafish; most serotonin-related genes were significantly increased in the transgenic zebrafish brains (Figure 3B), such as plasmacytoma expressed transcript 1 (pet1), tryptophan hydroxylase 2 (tph2) except solute carrier family 6, and member $4 a$ (slc6a4a). These data showed that the mRNA expression of the serotonin-related genes was upregulated following the overexpression of $s p x 1$ in the $\mathrm{dHb}$. Taken together, these data suggest that $s p x 1$ overexpression in the $\mathrm{dHb}$ partially reduced the anxiety response via the modulation of serotonin-related genes in the raphe.

\section{DISCUSSION}

This study is the first to reveal that the function of habenular SPX1 is associated with the regulation of the anxiety response. Overexpression of $s p x 1$ in the $\mathrm{dHb}$ reduced anxiety and upregulated galr $2 a$ and galr $2 b$ genes in the IPN and serotonin-related genes in the raphe. Although, we could not rule out the possibility of exogenous SPX1 expression in the $\mathrm{dHb}$ SPX1-negative neurons, which is caused by the Tg(gpr151:gal4vp16) driver line, our results are consistent with the earlier observation that the administration of an SPX-based GALR2-specific agonist induces anti-depressive and anxiolytic effects in mice (Reyes-Alcaraz et al., 2016; Yun et al., 2019), and that Galr2/3 knockout mice expressed an anxiety and depression-like phenotype (Bailey et al., 2007; Lu et al., 2008; Brunner et al., 2014). These data collectively suggest that $\mathrm{dHb}$ SPX1 has an anxiolytic function in zebrafish.

Several previous studies have provided indirect evidence suggesting that SPX1 function is implicated in anxiety. First, SPX1 has the capacity to bind Galr2 and Galr3 (Kim et al., 2014), which are implicated in anxiety and depression-like behaviors in mice (Bailey et al., 2007; Lu et al., 2008; Brunner et al., 2014). Second, administration of an SPX-based GALR2-specific agonist induces anti-depressive and anxiolytic effects in mice (ReyesAlcaraz et al., 2016; Yun et al., 2019). Third, habenula-IPN circuitry is implicated in fear and anxiety responses (Agetsuma et al., 2010; Hikosaka, 2010; Mathuru and Jesuthasan, 2013), and spx 1 is expressed in the specific nuclei of $\mathrm{dHb}$, which projects to the IPN in zebrafish (Kim et al., 2019). However, there is no direct evidence that SPX1 is functionally implicated in anxiety.

Recently, we have shown that SPX1 neurons are located in the $\mathrm{dHb}$ and project into the IPN, in which galr $2 a$ and galr $2 b$ were expressed, suggesting that $\mathrm{dHb}$ SPX 1 neurons may interact with GALR2a or GALR2b neurons in the IPN. Further, SPX1-GALR2a/2b neuronal circuits may be involved in the regulation of anxiety (Kim et al., 2019). A recent study has shown that intracerebroventricular administration of SPX1 increased the expression of Galr2 and Galr3 mRNA in responses to different types of pain, suggesting that SPX1 stimulates Galr2 or Galr3 expression and mediates anti-nociception ( $\mathrm{Lv}$ et al., 2019). Therefore, we hypothesized that the anxiolytic function of SPX1 in transgenic zebrafish overexpressing SPX1 in the $\mathrm{dHb}$ might be mediated by GALR2a or GALR2b in the IPN. Consistent with our hypothesis, we observed increased expression of galr $2 a$ and galr $2 b$ upon overexpression of spx1 in the $\mathrm{dHb}$, suggesting that $\mathrm{dHb}$ SPX1 neurons can regulate anxiety via interactions with GALR2a and GALR2b in the IPN.

Serotonin-related genes in the raphe were upregulated in transgenic zebrafish overexpressing spxi in the $\mathrm{dHb}$. The serotonergic system in the raphe is a major target of the Hb-IPN circuitry in mammals and fish (Herkenham and Nauta, 1979; Okamoto et al., 2012; Chou et al., 2016) and is involved in depression and anxiety (Ressler and Nemeroff, 2000; McDevitt et al., 2011). Previous studies have shown that Galr2 and Galr3 are expressed in the dorsal raphe nucleus (DRN; O’Donnell et al., 1999; Mennicken et al., 2002). In addition, a Galr2 agonist activated the DRN and increased the concentration of serotonin in rats (Mazarati et al., 2005), and Galr3 antagonists attenuated the inhibitory effect on serotonin neurotransmission in mice (Swanson et al., 2005), suggesting that Galr2 and Galr3 in the DRN directly modulate serotonergic neurons. However, our data suggested that SPX1-GALR2a/b mediated $\mathrm{Hb}$-IPN circuits may modulate raphe serotonergic system via galr2a/b activation in the IPN. Taken together, our data suggest that SPX1-Galr2a/b in Hb-IPN circuits are implicated in the anxiety response via regulation of the serotonergic system in the raphe. 


\section{DATA AVAILABILITY}

All datasets generated for this study are included in the manuscript and/or the Supplementary Files.

\section{ETHICS STATEMENT}

All experimental procedures were approved by the Korea University Institutional Animal Care and Use Committee (IACUC) and performed in accordance with Animal experiment guidelines of Korea National Veterinary Research and Quarantine Service.

\section{AUTHOR CONTRIBUTIONS}

IJ, EK and H-CP designed the study, analyzed the data, and wrote the manuscript. EK and IJ performed the experiments.

\section{REFERENCES}

Agetsuma, M., Aizawa, H., Aoki, T., Nakayama, R., Takahoko, M., Goto, M., et al. (2010). The habenula is crucial for experience-dependent modification of fear responses in zebrafish. Nat. Neurosci. 13, 1354-1356. doi: 10.1038/nn.2654

Aizawa, H., Bianco, I. H., Hamaoka, T., Miyashita, T., Uemura, O., Concha, M. L., et al. (2005). Laterotopic representation of left-right information onto the dorso-ventral axis of a zebrafish midbrain target nucleus. Curr. Biol. 15, 238-243. doi: 10.1016/j.cub.2005.01.014

Amo, R., Aizawa, H., Takahoko, M., Kobayashi, M., Takahashi, R., Aoki, T., et al. (2010). Identification of the zebrafish ventral habenula as a homolog of the mammalian lateral habenula. J. Neurosci. 30, 1566-1574. doi: 10.1523/JNEUROSCI.3690-09.2010

Bailey, K. R., Pavlova, M. N., Rohde, A. D., Hohmann, J. G., and Crawley, J. N. (2007). Galanin receptor subtype 2 (GalR2) null mutant mice display an anxiogenic-like phenotype specific to the elevated plus-maze. Pharmacol. Biochem. Behav. 86, 8-20. doi: 10.1016/j.pbb.2006.11.024

Broms, J., Antolin-Fontes, B., Tingstrom, A., and Ibanez-Tallon, I. (2015). Conserved expression of the GPR151 receptor in habenular axonal projections of vertebrates. J. Comp. Neurol. 523, 359-380. doi: 10.1002/cne.23664

Brunner, S. M., Farzi, A., Locker, F., Holub, B. S., Drexel, M., Reichmann, F., et al. (2014). GAL3 receptor KO mice exhibit an anxiety-like phenotype. Proc. Natl. Acad. Sci. U S A 111, 7138-7143. doi: 10.1073/pnas.1318066111

Cachat, J., Stewart, A., Grossman, L., Gaikwad, S., Kadri, F., Chung, K. M., et al. (2010). Measuring behavioral and endocrine responses to novelty stress in adult zebrafish. Nat. Protoc. 5, 1786-1799. doi: 10.1038/nprot.2010.140

Chou, M. Y., Amo, R., Kinoshita, M., Cherng, B. W., Shimazaki, H., Agetsuma, M., et al. (2016). Social conflict resolution regulated by two dorsal habenular subregions in zebrafish. Science 352, 87-90. doi: 10.1126/science.aac9508

Herkenham, M., and Nauta, W. J. (1979). Efferent connections of the habenular nuclei in the rat. J. Comp. Neurol. 187, 19-47. doi: 10.1002/cne.901870103

Hikosaka, O. (2010). The habenula: from stress evasion to value-based decisionmaking. Nat. Rev. Neurosci. 11, 503-513. doi: 10.1038/nrn2866

Hsu, Y. W., Morton, G., Guy, E. G., Wang, S. D., and Turner, E. E. (2016). Dorsal medial habenula regulation of mood-related behaviors and primary reinforcement by tachykinin-expressing habenula neurons. eNeuro 3:ENEURO.0109-16.2016. doi: 10.1523/eneuro.0109-16.2016

Kim, E., Jeong, I., Chung, A. Y., Kim, S., Kwon, S. H., Seong, J. Y., et al. (2019). Distribution and neuronal circuit of spexin 1/2 neurons in the zebrafish CNS. Sci. Rep. 9:5025. doi: 10.1038/s41598-019-41431-7

Kim, J. H., Lee, S. R., Li, L. H., Park, H. J., Park, J. H., Lee, K. Y., et al. (2011). High cleavage efficiency of a $2 \mathrm{~A}$ peptide derived from porcine teschovirus- 1 in human cell lines, zebrafish and mice. PLoS One 6:e18556. doi: 10.1371/journal. pone.0018556
JS advised and contributed to manuscript editing. All authors reviewed the manuscript.

\section{FUNDING}

This work was supported by Basic Science Research Program through the National Research Foundation of Korea (NRF) funded by the Ministry of Education (NRF2016R1D1A1B03935123) and by the Collaborative Genome Program of the Korea Institute of Marine Science and Technology Promotion (KIMST) funded by the Ministry of Oceans and Fisheries (MOF; No. 20180430).

\section{SUPPLEMENTARY MATERIAL}

The Supplementary Material for this article can be found online at: https://www.frontiersin.org/articles/10.3389/fncir.2019.000 53/full\#supplementary-material

Kim, D. K., Yun, S., Son, G. H., Hwang, J. I., Park, C. R., Kim, J. I., et al. (2014). Coevolution of the spexin/galanin/kisspeptin family: spexin activates galanin receptor type II and III. Endocrinology 155, 1864-1873. doi: 10.1210/en. 2013-2106

Liu, Y., Li, S., Qi, X., Zhou, W., Liu, X., Lin, H., et al. (2013). A novel neuropeptide in suppressing luteinizing hormone release in goldfish, Carassius auratus. Mol. Cell. Endocrinol. 374, 65-72. doi: 10.1016/j.mce.2013.04.008

Lu, X., Ross, B., Sanchez-Alavez, M., Zorrilla, E. P., and Bartfai, T. (2008). Phenotypic analysis of GalR2 knockout mice in anxiety- and depressionrelated behavioral tests. Neuropeptides 42, 387-397. doi: 10.1016/j.npep.2008. 04.009

Lv, S. Y., Cui, B., Yang, Y., Du, H., Zhang, X., Zhou, Y., et al. (2019). Spexin/NPQ induces FBJ osteosarcoma oncogene (Fos) and produces antinociceptive effect against inflammatory pain in the mouse model. Am. J. Pathol. 189, 886-899. doi: 10.1016/j.ajpath.2018.12.009

Ma, A., Bai, J., He, M., and Wong, A. O. L. (2018). Spexin as a neuroendocrine signal with emerging functions. Gen. Comp. Endocrinol. 265, 90-96. doi: 10.1016/j.ygcen.2018.01.015

Mathuru, A. S., and Jesuthasan, S. (2013). The medial habenula as a regulator of anxiety in adult zebrafish. Front. Neural Circuits 7:99. doi: 10.3389/fncir.2013. 00099

Mazarati, A. M., Baldwin, R. A., Shinmei, S., and Sankar, R. (2005). In vivo interaction between serotonin and galanin receptors types 1 and 2 in the dorsal raphe: implication for limbic seizures. J. Neurochem. 95, 1495-1503. doi: 10.1111/j.1471-4159.2005.03498.x

McDevitt, R. A., Hiroi, R., Mackenzie, S. M., Robin, N. C., Cohn, A., Kim, J. J., et al. (2011). Serotonin 1B autoreceptors originating in the caudal dorsal raphe nucleus reduce expression of fear and depression-like behavior. Biol. Psychiatry 69, 780-787. doi: 10.1016/j.biopsych.2010.12.029

Mennicken, F., Hoffert, C., Pelletier, M., Ahmad, S., and O'Donnell, D. (2002). Restricted distribution of galanin receptor 3 (GalR3) mRNA in the adult rat central nervous system. J. Chem. Neuroanat. 24, 257-268. doi: 10.1016/s08910618(02)00068-6

Mirabeau, O., Perlas, E., Severini, C., Audero, E., Gascuel, O., Possenti, R., et al. (2007). Identification of novel peptide hormones in the human proteome by hidden Markov model screening. Genome Res. 17, 320-327. doi: 10.1101/gr. 5755407

O'Donnell, D., Ahmad, S., Wahlestedt, C., and Walker, P. (1999). Expression of the novel galanin receptor subtype GALR2 in the adult rat CNS: distinct distribution from GALR1. J. Comp. Neurol. 409, 469-481. doi: 10.1002/(sici)1096-9861(19990705)409:3<469::aid-cne10> 3.3.co;2-h

Okamoto, H., Agetsuma, M., and Aizawa, H. (2012). Genetic dissection of the zebrafish habenula, a possible switching board for selection of behavioral 
strategy to cope with fear and anxiety. Dev. Neurobiol. 72, 386-394. doi: 10.1002/dneu.20913

Pandey, S., Shekhar, K., Regev, A., and Schier, A. F. (2018). Comprehensive identification and spatial mapping of habenular neuronal types using single-cell RNA-Seq. Curr. Biol. 28, 1052.e7-1065.e7. doi: 10.1016/j.cub.2018. 02.040

Porzionato, A., Rucinski, M., Macchi, V., Stecco, C., Malendowicz, L. K., and De Caro, R. (2010). Spexin expression in normal rat tissues. J. Histochem. Cytochem. 58, 825-837. doi: 10.1369/jhc.2010.956300

Ressler, K. J., and Nemeroff, C. B. (2000). Role of serotonergic and noradrenergic systems in the pathophysiology of depression and anxiety disorders. Depress. Anxiety 12, 2-19. doi: 10.1002/1520-6394(2000)12:1+<2::aid-da2>3. 3.co; $2-\mathrm{w}$

Reyes-Alcaraz, A., Lee, Y. N., Son, G. H., Kim, N. H., Kim, D. K., Yun, S., et al. (2016). Development of spexin-based human galanin receptor type II-specific agonists with increased stability in serum and anxiolytic effect in mice. Sci. Rep. 6:21453. doi: 10.1038/srep21453

Rucinski, M., Porzionato, A., Ziolkowska, A., Szyszka, M., Macchi, V., De Caro, R., et al. (2010). Expression of the spexin gene in the rat adrenal gland and evidences suggesting that spexin inhibits adrenocortical cell proliferation. Peptides 31, 676-682. doi: 10.1016/j.peptides.2009.12.025

Sonmez, K., Zaveri, N. T., Kerman, I. A., Burke, S., Neal, C. R., Xie, X., et al. (2009). Evolutionary sequence modeling for discovery of peptide hormones. PLoS Comput. Biol. 5:e1000258. doi: 10.1371/journal.pcbi.1000258

Swanson, C. J., Blackburn, T. P., Zhang, X., Zheng, K., Xu, Z. Q., Hökfelt, T., et al. (2005). Anxiolytic- and antidepressant-like profiles of the galanin-3 receptor (Gal3) antagonists SNAP 37889 and SNAP 398299. Proc. Natl. Acad. Sci. U S A 102, 17489-17494. doi: 10.1073/pnas.0508970102
Thisse, C., and Thisse, B. (2008). High-resolution in situ hybridization to wholemount zebrafish embryos. Nat. Protoc. 3, 59-69. doi: 10.1038/nprot.2007.514

Toll, L., Khroyan, T. V., Sonmez, K., Ozawa, A., Lindberg, I., McLaughlin, J. P., et al. (2012). Peptides derived from the prohormone proNPQ/spexin are potent central modulators of cardiovascular and renal function and nociception. FASEB J. 26, 947-954. doi: 10.1096/fj.11-192831

Wong, M. K., Sze, K. H., Chen, T., Cho, C. K., Law, H. C., Chu, I. K., et al. (2013). Goldfish spexin: solution structure and novel function as a satiety factor in feeding control. Am. J. Physiol. Endocrinol. Metab. 305, E348-E366. doi: 10.1152/ajpendo.00141.2013

Yamaguchi, T., Danjo, T., Pastan, I., Hikida, T., and Nakanishi, S. (2013). Distinct roles of segregated transmission of the septo-habenular pathway in anxiety and fear. Neuron 78, 537-544. doi: 10.1016/j.neuron.2013.02.035

Yun, S., Reyes-Alcaraz, A., Lee, Y. N., Yong, H. J., Choi, J., Ham, B. J., et al. (2019). Spexin-based galanin receptor type 2 agonist for comorbid mood disorders and abnormal body weight. Front. Neurosci. 13:391. doi: 10.3389/fnins.2019.00391

Conflict of Interest Statement: The authors declare that the research was conducted in the absence of any commercial or financial relationships that could be construed as a potential conflict of interest.

Copyright (c) 2019 Jeong, Kim, Seong and Park. This is an open-access article distributed under the terms of the Creative Commons Attribution License (CC BY). The use, distribution or reproduction in other forums is permitted, provided the original author(s) and the copyright owner(s) are credited and that the original publication in this journal is cited, in accordance with accepted academic practice. No use, distribution or reproduction is permitted which does not comply with these terms. 